\section{Associação entre aspectos psicossociais e excesso de peso referido em adultos de um município de médio porte do Sul do Brasil}

\author{
Association between psychosocial factors and \\ self-reported overweight in Brazilian adults
}

1. Programa de Pós-graduação
em Saúde Coletiva,
Universidade do Vale do Rio
dos Sinos, São Leopoldo,
Brasil.
Correspondência
M. P. Pattussi
Programa de Pós-graduação
em Saúde Coletiva,
Universidade do Vale do Rio
dos Sinos.
Av. Unisinos 950, C. P. 275, São
Leopoldo, RS 93022-000, Brasil.
mppattussi@unisinos.br

Abstract

The objective of this study was to investigate the association between psychosocial factors and self-reported overweight. A cross-sectional study was carried out with 1,100 adults in a Brazilian city. Structured interviews were conducted using a standard pre-tested questionnaire. Overweight was self-reported by participants and defined as body mass index (BMI) $\geq 25 \mathrm{~kg} / \mathrm{m}^{2}$. Psychosocial factors were quality of life (WHO-8: EUROHIS) and social support (Medical Outcomes Study). Crude and adjusted prevalence ratios (PR) were obtained by modified Poisson regression. After controlling for socio-demographic and behavioral variables, prevalence of overweight among individuals reporting low quality of life and low social support was 19\% (PR = 1.19; 95\%CI: 1.031.38) and 12\% (PR = 1.12; 95\%CI: 0.96-1.31), respectively, and was higher in those reporting high/ moderate quality of life and high/moderate social support, respectively. The findings suggest that psychosocial factors can play a role in self-assessment of overweight.

Overweight; Social Support; Quality of Life; Adult
Vanessa Backes 1 Maria Teresa Anselmo Olinto 1 Ruth Liane Henn 1 Cleber Cremonese 1 Marcos Pascoal Pattussi 1

\section{Introdução}

Dentre as doenças crônicas não transmissíveis, a obesidade é um dos mais preocupantes problemas de saúde pública do mundo, haja vista os agravos dela decorrentes e o consequente aumento nos custos sociais da atenção à saúde 1,2 . Os problemas crônicos associados à obesidade residem em quatro áreas principais: problemas cardiovasculares; condições associadas à resistência insulínica; colecistopatias e certos tipos de câncer ${ }^{3}$. Quando não fatais, essas enfermidades podem ser debilitantes, comprometendo a qualidade de vida e implicando custos sociais e econômicos crescentes 4 .

Os fatores de risco para o excesso de peso são bem estabelecidos e incluem os fatores socioeconômicos, demográficos, genéticos, culturais, comportamentais e fisiológicos 3 . Além destes, aspectos psicossociais parecem estar associados com o excesso de peso ${ }^{5}$. Estados psicológicos como autoestima, autoeficácia e segurança influenciariam comportamentos relacionados à saúde, tais como o hábito de fumar, consumo de álcool e alimentação ${ }^{6}$. Por outro lado, a imagem negativa presente nos obesos provoca sofrimento e sérias dificuldades no âmbito social e ocupacional, incapacitação física, absenteísmo, aumento de licenças médicas, perda e/ou recusa de emprego, queda de renda, ansiedade, baixa autoestima e isolamento social 7 . 
O presente estudo tem como objetivo explorar a associação entre aspectos psicossociais excesso de peso referido em adultos.

\section{Metodologia}

Foi realizado um estudo transversal de base populacional, com uma amostra representativa de adultos residentes na zona urbana do Município de São Leopoldo, Rio Grande do Sul, Brasil. O município está situado na região do Vale do Rio dos Sinos, Região Metropolitana de Porto Alegre; conta, na sua estrutura de serviços públicos de saúde, com um hospital geral e 28 unidades básicas de saúde. No ano de 2000, apresentava população de aproximadamente 194 mil habitantes (Instituto Brasileiro de Geografia e Estatística. Cidades@.http://www.ibge.gov.br/cidadesat/ topwindow.htm?1).

Os dados foram coletados por meio de entrevistas estruturadas com os responsáveis pelos domicílios, utilizando-se um questionário padronizado e pré-testado, composto por variáveis demográficas, socioeconômicas, comportamentais e psicossociais. Um estudo-piloto com cem pessoas serviu como base para avaliar a qualidade dos instrumentos, além de apreciar os métodos e a logística da pesquisa.

O cálculo do tamanho da amostra foi feito com base nos dados do estudo-piloto, utilizandose o método para proporções com aleatorização de conglomerados ${ }^{8}$ do desfecho autopercepção em saúde. Estimou-se ser necessária amostra de 1.260 domicílios em 36 setores. Considerou-se poder de $85 \%(1-\beta=0.85)$ para se detectar diferença de $7 \%$ na prevalência de autopercepção de saúde ruim entre áreas com alto e baixo capital social, com nível de 95\% de confiança. Assumindo-se um número de 35 domicílios por setor e coeficiente de correlação intraclasse igual a 0,05, o tamanho da amostra foi aumentado em $20 \%$ no número de domicílios e em 10\%, no número de setores, em virtude de possíveis perdas e a fim de controlar fatores de confusão na análise dos dados. Para isso, foi necessário um total de 1.512 domicílios em quarenta setores censitários.

A seleção se deu em dois estágios. Primeiro, foi realizado um sorteio aleatório dos setores censitários dentre os 270 existentes na zona urbana da cidade. Em seguida, sortearam-se quadras dentro dos setores, e todos os domicílios foram visitados até completar o número requerido de 38. Se, ao completar o quarteirão, não houvesse o número suficiente de residências, o entrevistador percorria outro quarteirão do setor, seguindo sorteio prévio. Caso houvesse mais domicílios do que o necessário naquele quarteirão, sorteava- se, aleatoriamente, a esquina, a partir da qual o domicilio era identificado para o início da coleta de dados. O início se dava pela esquerda de quem estivesse de costas para a casa inicial; mantendose o sentido anti-horário, o domicílio seguinte era visitado e o próximo entrava na seleção. Em caso de ausência do responsável pelo domicílio, realizavam-se três visitas de retorno.

O desfecho do estudo foi avaliado por intermédio do índice da massa corporal (IMC: razão entre o peso em quilogramas e o quadrado da estatura, em metros), sendo o peso e a altura referidos pelo entrevistado. Foram considerados com excesso de peso os indivíduos que apresentaram $\mathrm{IMC} \geq 25 \mathrm{~kg} / \mathrm{m}^{2} 9$.

As variáveis psicossociais utilizadas no presente estudo foram qualidade de vida e apoio social.

Qualidade de vida foi avaliada com a escala WHO-8:EUROHIS, composta por oito itens derivados da versão curta do WHOQOL, os quais versam sobre a satisfação do entrevistado consigo mesmo, com os relacionamentos pessoais, com a renda, com as condições de moradia, com a saúde, com o desempenho em atividades diárias, e apresentam a sua opinião sobre qualidade de vida e energia para enfrentar o dia a dia 10 . Os itens foram adaptados para o português baseados na versão brasileira do WHOQOL-Bref 11. As respostas a esses itens foram medidas através de uma escala de cinco pontos, variando de "muito insatisfeito" a "muito satisfeito". Na análise, a escala variou de 0 a 4 , e considerou-se a soma de todas as respostas, tendo como valor mínimo possível 0 e valor máximo, 32. Os maiores escores representando maior qualidade de vida. Esta variável foi categorizada em alta $(25 \%$ dos escores mais elevados), moderada (50\% dos escores intermediários) e baixa qualidade de vida (25\% dos escores menores). As categorias alta e moderada foram unidas porque o tamanho de seus efeitos não diferiu. A escala foi validada em dez países da Europa por Schmidt et al. 12. No Brasil, os dados de reprodutibilidade e validade referem-se à versão curta do WHOQOL 11 .

A variável apoio social foi coletada por meio da escala de apoio social utilizada no Medical Outcomes Study (MOS) 13. Esta escala é composta por 19 itens, compreendendo cinco dimensões funcionais: material, afetivo, emocional, interação social positiva e informação. Para todos os itens, cinco opções de resposta, variando de "nunca" a "sempre", foram apresentadas. Na análise, a escala variou de 0 a 4 , sendo utilizado o somatório das respostas, cujo resultado poderia variar de 0 a 76; os valores elevados representando maior apoio social. A categorização adotou o mesmo princípio da qualidade de vida, e a variá- 
vel foi dicotomizada em alta/moderada e baixa. A escala foi validada no Brasil por Griep et al. 14,15.

As variáveis demográficas incluíam sexo, idade (grupos de 10 anos), cor da pele (branca e não branca) e estado civil (casado/união, solteiro e outra situação). As variáveis socioeconômicas individuais foram renda familiar (em reais) e escolaridade (anos de estudo), posteriormente categorizadas em alta (25\% dos maiores escores dentre os indivíduos), média (50\% dos escores intermediários) e baixa (25\% dos escores menores). As variáveis comportamentais foram hábito de fumar (nunca fumou, ex-fumante e fumante), atividade física [sedentário: não pratica atividade física ou pratica menos de 150 minutos/semana; não sedentário: ativo regular e/ou muito ativo ( $\geq 150$ minutos/semana)] 16 e consumo de álcool [não consome/consome moderadamente (<15mg 우 e < 30mg đ̋); sim/excesso ( $\geq 15 \mathrm{mg}$ 우 $\left.\left.\mathrm{e} \geq 30 \mathrm{mg} \delta^{\Uparrow}\right)\right] 17$.

O controle de qualidade da coleta de dados foi feito por telefone, em uma amostra aleatória de $10 \%$ das pessoas participantes do estudo. $\mathrm{O}$ instrumento do controle era semelhante ao do estudo, incluindo variáveis que não sofriam alteração em curto espaço de tempo.

A entrada de dados foi realizada no programa Epi Info 6, versão 6.0 (Centers for Disease Control and Prevention, Atlanta, Estados Unidos), em dupla entrada e posterior comparação, para se eliminar a probabilidade de erros de digitação. A análise dos dados foi conduzida no programa Stata 9.0 (Stata Corp., College Station, Estados Unidos). Para fornecer uma estimativa das razões de prevalências (RP) brutas e ajustadas, além de seus respectivos intervalos de $95 \%$ de confiança (IC95\%), utilizou-se a regressão de Poisson, com controle para efeito de delineamento.

Apenas os possíveis fatores de confusão entraram na análise multivariável. Para ser considerada como fator de confusão, a variável deveria estar associada tanto com a exposição quanto com o desfecho, considerando um nível de significância menor que $20 \%(\mathrm{p}<0,20)$. A análise multivariável foi realizada mediante três modelos. No primeiro, o efeito da variável psicossocial foi controlado para características demográficas; no segundo, incluíram-se as variáveis socioeconômicas; no terceiro, as comportamentais.

O protocolo de pesquisa foi aprovado pelo Comitê de Ética em Pesquisa da Universidade do Vale do Rio dos Sinos (UNISINOS; CEP 04/034). Foi requerido o consentimento livre e esclarecido dos participantes, aos quais foi garantido o total sigilo dos dados.

\section{Resultados}

Do total previsto, foram realizadas 1.100 entrevistas em 38 setores. Em 1.058 destas, foram referidos peso e altura. Locais não habitados ou comércio representaram $8 \%$; recusas, $4 \%$; e pessoas responsáveis pelas residências ausentes no momento da entrevista, $2 \%$. Com base nos dados referidos, encontrou-se prevalência de excesso de peso referido de 48,6\% (IC95\%: 44,9-52,3).

A maioria da população estudada eram mulheres $(71,8 \%)$, de cor branca $(84 \%)$, casadas (56\%), com idade entre 30 e 49 anos (40\%), ex-fumantes $(57,1 \%)$, que apresentavam renda familiar média de 7,5 ( $\mathrm{DP}=7,4)$ e escolaridade média de $8(\mathrm{DP}=4)$. Maiores prevalências de excesso de peso $(\mathrm{p}<0,05)$ foram encontradas nas faixas etárias mais elevadas, em pessoas com baixa escolaridade e com baixa qualidade de vida (Tabela 1 ).

$\mathrm{Na}$ análise bruta, indivíduos relatando baixa qualidade de vida apresentavam prevalência $29 \%$ mais elevada de excesso de peso, quando comparados com aqueles com alta/moderada qualidade de vida (RP = 1,29; IC95\%: 1,05-1,57). Após o controle por sexo, faixa etária, estado civil, escolaridade, hábito de fumar e atividade física (modelo 4), a associação entre baixa qualidade de vida e excesso de peso manteve-se $(\mathrm{RP}=1,19$; IC95\%: 1,03-1,38) (Tabela 2). Quanto ao apoio social, após ajuste por variáveis demográficas, socioeconômicas e comportamentais, observou-se prevalência $12 \%$ mais elevada de excesso de peso em pessoas com baixo apoio, quando comparadas àquelas com alto/moderado apoio social $(\mathrm{RP}=1,12$; IC95\%: 0,96-1,31). Embora sem significância estatística, a magnitude da associação entre apoio social e excesso de peso, nos diferentes modelos, mostrou tendência similar (Tabela 3).

\section{Discussão}

Este estudo teve como objetivo investigar a associação entre aspectos psicossociais e excesso de peso. Nossos resultados mostraram que baixa qualidade de vida esteve associada ao excesso de peso, mesmo após ajuste por variáveis demográficas, socioeconômicas e comportamentais.

Medidas de qualidade de vida avaliam aspectos importantes de saúde que não são detectados pelas dimensões fisiológicas e clínicas tradicionais 18 . Umas das possíveis causas da baixa qualidade de vida em indivíduos com excesso de peso é que estes apresentam diversas limitações físicas e emocionais. Estudos de base populacional demonstram que essas pessoas frequentemente queixam-se de dor, relatam redução da vitalidade, funcionamento físico deficiente, 
Distribuição da amostra de acordo com variáveis socioeconômicas, demográficas, psicossociais, comportamentais, e prevalência de excesso de peso referido em adultos. São Leopoldo, Rio Grande do Sul, Brasil, 2007 ( $n=1.058$ ).

\begin{tabular}{|c|c|c|c|c|c|}
\hline \multirow[t]{2}{*}{ Variáveis } & \multirow[t]{2}{*}{$\mathrm{n}$} & \multirow[t]{2}{*}{$\%$} & \multicolumn{2}{|c|}{$\begin{array}{c}\text { Prevalência de excesso de } \\
\text { peso }\end{array}$} & \multirow[t]{2}{*}{ Valor de $p$} \\
\hline & & & $\%$ & IC95\% & \\
\hline Sexo & & & & & 0,060 \\
\hline Masculino & 310 & 28,2 & 53,1 & $47,5-58,6$ & \\
\hline Feminino & 790 & 71,8 & 46,7 & $43,2-50,3$ & \\
\hline Cor da pele & & & & & 0,673 \\
\hline Branca & 922 & 84,0 & 48,2 & $44,9-51,5$ & \\
\hline Não branca & 176 & 16,0 & 50,0 & $42,4-57,6$ & \\
\hline Faixa etária (anos) & & & & & $<0,001$ \\
\hline $18-29$ & 247 & 22,5 & 31,1 & $25,6-37,2$ & \\
\hline $30-39$ & 182 & 16,5 & 41,3 & $34,4-48,7$ & \\
\hline $40-49$ & 258 & 23,5 & 54,0 & $47,8-60,1$ & \\
\hline $50-59$ & 217 & 19,7 & 54,9 & $48,0-61,5$ & \\
\hline$\geq 60$ & 196 & 17,8 & 63,6 & $56,5-70,2$ & \\
\hline Estado civil & & & & & $<0,001$ \\
\hline Casado/União & 615 & 55,9 & 53,2 & $49,2-57,2$ & \\
\hline Solteiro & 267 & 24,3 & 35,4 & $29,8-41,4$ & \\
\hline Outra & 218 & 19,8 & 51,7 & $44,9-58,5$ & \\
\hline Escolaridade * & & & & & $<0,001$ \\
\hline Alta & 166 & 18,0 & 39,4 & $32,3-47,0$ & \\
\hline Média & 552 & 59,9 & 46,8 & $43,9-51,5$ & \\
\hline Baixa & 204 & 22,1 & 61,7 & $54,6-68,4$ & \\
\hline Renda familiar ** & & & & & 0,971 \\
\hline Alta & 268 & 25,1 & 49,3 & $43,2-55,3$ & \\
\hline Média & 565 & 53,0 & 48,1 & $43,8-52,3$ & \\
\hline Baixa & 234 & 21,9 & 49,8 & $43,1-56,4$ & \\
\hline Consumo de álcool *** & & & & & 0,193 \\
\hline Não & 1.070 & 97,3 & 48,3 & $45,2-51,3$ & \\
\hline Sim & 30 & 2,7 & 60,7 & $42,7-76,4$ & \\
\hline Atividade física \# & & & & & 0,106 \\
\hline Não sedentário & 191 & 17,4 & 43,3 & $36,4-50,5$ & \\
\hline Sedentário & 907 & 82,6 & 49,8 & $46,5-53,1$ & \\
\hline Hábito de fumar & & & & & $<0,001$ \\
\hline Nunca fumou & 605 & 55,0 & 49,9 & $45,6-54,0$ & \\
\hline Ex-fumante & 233 & 21,2 & 57,1 & $50,6-63,4$ & \\
\hline Fumante & 262 & 23,8 & 37,8 & $32,1-44,0$ & \\
\hline Apoio social & & & & & 0,066 \\
\hline Alto/Moderado & 806 & 76,5 & 47,0 & $43,6-50,5$ & \\
\hline Baixo & 247 & 23,5 & 53,8 & $47,6-60,0$ & \\
\hline Qualidade de vida & & & & & $<0,001$ \\
\hline Alta/Moderada & 833 & 79,3 & 45,9 & $42,5-49,3$ & \\
\hline Baixa & 217 & 20,7 & 59,0 & $52,3-65,3$ & \\
\hline
\end{tabular}

Nota: valores de $n$ total diferentes em cada variável referem-se a casos ausentes (missing information).

* Em anos de estudo: alta (25\% dos maiores escores dentre os indivíduos), média (50\% dos escores intermediários) e baixa (25\% dos escores menores);

** Em reais: alta (25\% dos maiores escores dentre os indivíduos), média (50\% dos escores intermediários) e baixa (25\% dos escores menores);

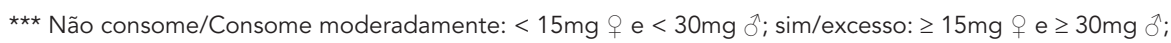

\# Sedentário: $<150$ minutos/semana; não sedentário: $\geq 150$ minutos/semana. 
Associação entre qualidade de vida e excesso de peso referido de acordo com os diversos modelos de ajuste.

\begin{tabular}{|c|c|c|c|c|}
\hline Variáveis * & Modelo 1 & Modelo 2 & Modelo 3 & Modelo 4 \\
\hline \multicolumn{5}{|l|}{ Qualidade de vida } \\
\hline Alta/Moderada & 1,00 & 1,00 & 1,00 & 1,00 \\
\hline Baixa & $1,29(1,05-1,57)$ & $1,22(1,06-1,41)$ & $1,18(1,03-1,35)$ & $1,19(1,03-1,38)$ \\
\hline \multicolumn{5}{|l|}{ Sexo } \\
\hline Feminino & & 1,00 & 1,00 & 1,00 \\
\hline Masculino & & $0,89(0,78-1,01)$ & $0,86(0,76-0,98)$ & $0,85(0,74-0,98)$ \\
\hline \multicolumn{5}{|l|}{ Faixa etária (anos) } \\
\hline $18-29$ & & 1,00 & 1,00 & 1,00 \\
\hline $30-39$ & & $1,26(1,00-1,59)$ & $1,27(1,00-1,60)$ & $1,29(1,02-1,62)$ \\
\hline $40-49$ & & $1,59(1,27-2,00)$ & $1,59(1,28-1,99)$ & $1,64(1,31-2,05)$ \\
\hline $50-59$ & & $1,59(1,24-2,04)$ & $1,55(1,20-2,01)$ & $1,61(1,23-2,01)$ \\
\hline$\geq 60$ & & $1,89(1,48-2,42)$ & $1,81(1,40-2,33)$ & $1,77(1,36-2,31)$ \\
\hline \multicolumn{5}{|l|}{ Estado civil } \\
\hline Casado/União & & 1,00 & 1,00 & 1,00 \\
\hline Solteiro & & $0,81(0,65-1,00)$ & $0,80(0,65-0,99)$ & $0,83(0,68-1,02)$ \\
\hline Outra & & $0,84(0,71-1,00)$ & $0,87(0,73-1,02)$ & $0,87(0,73-1,03)$ \\
\hline \multicolumn{5}{|l|}{ Escolaridade } \\
\hline Alta & & & 1,00 & 1,00 \\
\hline Média & & & $1,13(0,92-1,37)$ & $1,14(0,93-1,40)$ \\
\hline Baixa & & & $1,32(1,05-1,66)$ & $1,35(1,07-1,69)$ \\
\hline \multicolumn{5}{|l|}{ Atividade física } \\
\hline Não sedentário & & & & 1,00 \\
\hline Sedentário & & & & $1,08(0,96-1,22)$ \\
\hline \multicolumn{5}{|l|}{ Hábito de fumar } \\
\hline Nunca fumou & & & & 1,00 \\
\hline Ex-fumante & & & & $0,99(0,84-1,16)$ \\
\hline Fumante & & & & $0,73(0,62-0,86)$ \\
\hline
\end{tabular}

Modelo 1: efeito da qualidade de vida sem ajuste; modelo 2: qualidade de vida ajustada por variáveis demográficas; modelo 3: qualidade de vida ajustada por variáveis demográficas e socioeconômicas; modelo 4: qualidade de vida ajustada por variáveis demográficas, socioeconômicas e comportamentais. Variáveis de cada modelo estão ajustadas entre si.

* Variáveis associadas com o desfecho e com a exposição no nível de significância menor que $20 \%(p<0,2)$.

dificuldades nos papéis sociais ou profissionais e pior estado de saúde geral, quando comparadas com as não obesas 19,20,21,22,23. Richards et al. 24, utilizando o Medical Outcomes Study 36Item Short-Form Health Survey (SF-36), observaram que indivíduos com obesidade grave (IMC > $35 \mathrm{~kg} / \mathrm{m}^{2}$ ) apresentaram bem-estar emocional e status funcional prejudicados, além de dor mais grave e limitante, quando comparados com os eutróficos. Em adição, observa-se que obesos são mais expostos a situações humilhantes em decorrência de suas restrições físicas 25 . Outro aspecto a considerar neste processo é a presença de morbidades associadas ao excesso de peso, como, por exemplo, diabetes, hipertensão e depressão, as quais também influenciariam esta- dos físicos e mentais 24. Entretanto, Doll et al. 21 demonstraram que a obesidade e co-morbidades estavam independentemente relacionadas com a diminuição do funcionamento físico, sugerindo, assim, qualidade de vida diminuída em indivíduos com obesidade mórbida.

No presente estudo, também houve uma tendência de o excesso de peso estar associado com baixo apoio social. Essa tendência manteve-se independente da inclusão de outros fatores de confusão. Um dos possíveis mecanismos de ação seria a atuação do apoio social na saúde dos indivíduos por meio da promoção de hábitos saudáveis e redução de comportamentos de risco, ocasionando alterações nos sistemas imunológico, nervoso e cardiovascular 26. A provisão de 
Associação entre apoio social e excesso de peso referido de acordo com os diversos modelos de ajuste.

\begin{tabular}{|c|c|c|c|c|}
\hline Variáveis * & Modelo 1 & Modelo 2 & Modelo 3 & Modelo 4 \\
\hline \multicolumn{5}{|l|}{ Apoio social } \\
\hline Alto/Moderado & 1,00 & 1,00 & 1,00 & 1,00 \\
\hline Baixo & $1,14(0,94-1,39)$ & $1,15(1,00-1,32)$ & $1,12(0,97-1,30)$ & $1,12(0,96-1,31)$ \\
\hline \multicolumn{5}{|l|}{ Sexo } \\
\hline Feminino & & 1,00 & 1,00 & 1,00 \\
\hline Masculino & & $0,90(0,80-1,01)$ & $0,87(0,77-0,98)$ & $0,86(0,75-1,00)$ \\
\hline \multicolumn{5}{|l|}{ Faixa etária (anos) } \\
\hline $18-29$ & & 1,00 & 1,00 & 1,00 \\
\hline $30-39$ & & $1,26(1,00-1,60)$ & $1,27(1,01-1,61)$ & $1,29(1,03-1,62)$ \\
\hline $40-49$ & & $1,62(1,30-2,03)$ & $1,62(1,30-2,01)$ & $1,66(1,33-2,07)$ \\
\hline $50-59$ & & $1,64(1,28-2,11)$ & $1,60(1,23-2,07)$ & $1,66(1,28-2,16)$ \\
\hline$\geq 60$ & & $1,96(1,54-2,49)$ & $1,85(1,44-2,37)$ & $1,82(1,39-2,38)$ \\
\hline \multicolumn{5}{|l|}{ Estado civil } \\
\hline Casado/União & & 1,00 & 1,00 & 1,00 \\
\hline Solteiro & & $0,80(0,64-0,99)$ & $0,79(0,64-0,98)$ & $0,81(0,66-1,00)$ \\
\hline Outra & & $0,84(0,71-0,99)$ & $0,86(0,73-1,02)$ & $0,88(0,74-1,05)$ \\
\hline \multicolumn{5}{|l|}{ Escolaridade } \\
\hline Alta & & & 1,00 & 1,00 \\
\hline Média & & & $1,14(0,93-1,40)$ & $1,17(0,95-1,43)$ \\
\hline Baixa & & & $1,36(1,07-1,71)$ & $1,40(1,11-1,77)$ \\
\hline \multicolumn{5}{|l|}{ Hábito de fumar } \\
\hline Nunca fumou & & & & 1,00 \\
\hline Ex-fumante & & & & $0,97(0,83-1,14)$ \\
\hline Fumante & & & & $0,73(0,62-0,87)$ \\
\hline
\end{tabular}

Modelo 1: efeito do apoio social sem ajuste; modelo 2: apoio social ajustado por variáveis demográficas; modelo 3: apoio social ajustado por variáveis demográficas e socioeconômicas; modelo 4: apoio social ajustado por variáveis demográficas, socioeconômicas e comportamentais. Variáveis de cada modelo estão ajustadas entre si.

* Variáveis associadas com o desfecho e com a exposição no nível de significância menor que $20 \%(p<0,2)$.

recursos materiais, a disseminação de informações preventivas e as respostas diretas ao estresse fisiológico influenciariam estados psicológicos como autoestima, autoeficácia e segurança, interferindo, consequentemente, em comportamentos relacionados à saúde, tais como hábito de fumar, consumo de álcool, alimentação, utilização de serviços de saúde, aderência ao tratamento e prática de exercícios físicos 6,27.

Embora as análises tenham sido conduzidas apenas com o escore global da escala de apoio social, procurou-se entender melhor os resultados, investigando-se a relação de cada uma das dimensões com o desfecho. Os autores proponentes da escala 13 relatam a existência de cinco dimensões, porém, em nosso estudo, a análise fatorial exploratória identificou quatro dimensões, uma vez que "apoio emocional" e "informação” constituíram-se em apenas um fator. Entre as quatro dimensões, "apoio afetivo" foi a única a apresentar associação com excesso de peso. Sugere-se que outros estudos façam essa avaliação das dimensões de forma independente e, assim, contribuam para a compreensão do fenômeno que está sendo estudado.

Os resultados deste estudo, entretanto, devem ser discutidos considerando-se algumas limitações metodológicas. A primeira delas refere-se à utilização de peso e altura referidos. A literatura demonstra que pessoas obesas tendem a subestimar o seu peso, assim como pessoas de baixa estatura, a superestimar a sua altura 28,29. Isso significa que a prevalência de excesso de peso encontrada neste estudo pode estar subestimada, fato que implica a perda de poder para detectar associações. Por outro lado, estudos têm demonstrado níveis aceitáveis de validade e confiabilidade para obesidade referida 30,31. Além 
disso, o autorrelato é um importante método para coletar informações em saúde, entre adultos. Ele permite o uso de pessoal auxiliar, o que o torna ideal para intervenções coletivas e um bom indicador para o planejamento e monitoramento de intervenções.

Outra limitação do estudo diz respeito ao seu delineamento. Estudos com delineamento transversal são limitados à identificação de associações, não sendo possível estabelecer relações causais. Causalidade reversa pode existir, pois tanto exposição quanto desfecho são medidos no mesmo ponto no tempo 32 . Por exemplo, poder-se-ia argumentar que aspectos psicossociais desfavoráveis, por um lado, poderiam levar a uma alteração comportamental que influencia- ria o excesso de peso, mas, por outro, poderiam ser apenas consequências emocionais e físicas de um excesso de peso já instalado. Sendo assim, estudos longitudinais são essenciais para elucidar as associações aqui descritas.

Apesar das limitações, os achados deste trabalho têm relevância como fonte para futuras comparações por identificar associação entre a qualidade de vida e o excesso de peso, levando em consideração os aspectos comportamentais. Em termos de saúde pública, o estudo aponta para a necessidade de atuação interdisciplinar em programas e ações para o controle de peso, pois o excesso de peso é uma doença multifatorial, que envolve, além de aspectos biológicos, aspectos psicossociais e comportamentais.

\section{Resumo}

Estudo transversal de base populacional, com 1.100 adultos de São Leopoldo, Rio Grande do Sul, Brasil, investigou a associação entre aspectos psicossociais e excesso de peso. Entrevistas estruturadas foram realizadas utilizando-se questionário padronizado e prétestado. Dados antropométricos foram referidos pelos participantes, e excesso de peso foi definido pelo índice de massa corporal (IMC) $\geq 25 \mathrm{~kg} / \mathrm{m}^{2}$. Aspectos psicossociais incluíram qualidade de vida (WHO-8:EUROHIS) e apoio social (Medical Outcomes Study). A análise dos dados foi realizada com regressão de Poisson modificada. Após ajuste para variáveis sociodemográficas e comportamentais, as prevalências de excesso de peso foram 19\% (RP = 1,19; IC95\%: 1,03-1,38) e 12\% $(R P=1,12 ;$ IC95\% 0,96-1,31) mais elevadas em pessoas com, respectivamente, baixa qualidade de vida e baixo apoio sócial, quando comparadas àquelas com níveis altos ou moderados nestas características. Os achados sugerem que aspectos psicossociais podem apresentar influência no excesso de peso, indicando, assim, a necessidade da atuação interdisciplinar em programas e ações para o controle de peso.

Sobrepeso; Apoio Social; Qualidade de Vida; Adulto

\section{Colaboradores}

V. Backes planejou o estudo, participou da coleta e análise dos dados e redigiu o artigo. M. T. A. Olinto e R. L. Henn contribuíram na concepção e planejamento do estudo. C. Cremonese participou da coleta e análise dos dados. M. P. Pattussi coordenou todas as etapas do estudo, da concepção, delineamento, análise dos dados até a redação final do artigo. Todos os autores trabalharam na redação do artigo, revisaram criticamente e aprovaram a versão final.

\section{Agradecimentos}

V. Backes e C. Cremonese foram financiados pela Coordenação de Aperfeiçoamento de Pessoal de Nível Superior (CAPES), com bolsa PROSUP/CAPES. M. T. A. Olinto recebeu bolsa de produtividade do Conselho Nacional de Desenvolvimento Científico e Tecnológico (CNPq; processo 308833/2006-6). M. P. Pattussi recebeu auxílio financeiro do CNPq (processos 478503/2004-0 e 481410/2009-0) e da Fundação de Amparo à Pesquisa do Estado do Rio Grande do Sul (FAPERGS; processo 0415621). 


\section{Referências}

1. Gortmaker SL, Must A, Perrin JM, Sobol AM, Dietz $\mathrm{WH}$. Social and economic consequences of overweight in adolescence and young adulthood. N Engl J Med 1993; 329:1008-12.

2. Rössner S. Obesity: the disease of the twenty-first century. Int J Obes Relat Metab Disord 2002; 26 Suppl 4:S2-4.

3. Organização Mundial da Saúde. Obesidade: prevenindo e controlando a epidemia global: relatório da consultoria da OMS. São Paulo: Editora Roca; 2004.

4. Pereira LO, Francischi RP, Lancha Júnior AH. Obesidade: causas e conseqüências. Arq Bras Endocrinol Metab 2003; 47:111-27.

5. Pender JR, Pories WJ. Epidemiology of obesity in the United States. Gastroenterol Clin North Am 2005; 34:1-7.

6. Berkman LF, Glass T, Brissette I, Seeman TE. From social integration to health: Durkheim in the new millennium. Soc Sci Med 2000; 51:843-57.

7. Souza JMB, Castro MM, Maia EMC, Ribeiro AN, Almondes KM, Silva NG. Obesidade e tratamento: desafio comportamental e social. Rev Bras Ter Cogn 2005; 1:59-67.

8. Hsieh FY. Sample size formulae for intervention studies with the cluster as unit of randomization. Stat Med 1988; 7:1195-201.

9. Mendonça CP, Anjos LA. Aspectos das práticas alimentares e da atividade física como determinantes do crescimento do sobrepeso/obesidade no Brasil. Cad Saúde Pública 2004; 20:698-709.

10. Power M. Development of a common instrument for quality of life. In: Nosikov A, Gudex C, editors. EUROHIS: developing common instruments for health surveys. Amsterdam: IOS Press/World Health Organization Regional Office for Europe; 2003. p. 145-63. (Biomedical and Health Research).

11. Fleck MP, Louzada S, Xavier M, Chachamovich E, Vieira G, Santos L, et al. Aplicação da versão em português do instrumento abreviado de avaliação da qualidade de vida "WHOQOL-Bref". Rev Saúde Pública 2000; 34:178-83.

12. Schmidt S, Muhlan H, Power M. The EUROHISQOL 8-item index: psychometric results of a cross-cultural field study. Eur J Public Health 2006; 16:420-8.

13. Sherbourne CD, Stewart AL. The MOS social support survey. Soc Sci Med 1991; 32:705-14.

14. Griep RH, Chor D, Faerstein E, Lopes, C. Apoio social: confiabilidade teste-reteste de escala no Estudo Pró-Saúde. Cad Saúde Pública 2003; 19:625-34.

15. Griep RH, Chor D, Faerstein E, Werneck GL, Lopes CS. Validade de constructo de escala de apoio social do Medical Outcomes Study adaptada para o português no Estudo Pró-Saúde. Cad Saúde Pública 2005; 21:703-14.

16. Hallal PC, Victora CG. Reliability and validity of the International Physical Activity Questionnaire (IPAQ). Med Sci Sports Exerc 2004; 36:556.

17. Steffens AA, Moreira LB, Fuchs SC, Wiehe M, Gus $\mathrm{M}$, Fuchs FD. Incidence of hypertension by alcohol consumption: is it modified by race? J Hipertens 2006; 24:1489-92.
18. Kunkel N, Oliveira WF, Peres MA. Excesso de peso e qualidade de vida relacionada à saúde em adolescentes de Florianópolis, SC. Rev Saúde Pública 2009; 43:226-35.

19. Wadden TA, Sarwer DB, Womble LG, Foster GD, McGuckin BG, Schimmel A. Psychosocial aspects of obesity and obesity surgery. SURG Clin North Am 2001; 81:1001-24.

20. Le Pen C, Levy E, Loos F, Banzet MN, Basdevant A. "Specific" scale compared with "generic" scale: a double measurement of the quality of life in a French community sample of obese subjects. J Epidemiol Community Health 1998; 52:445-50.

21. Doll HA, Petersen SE, Stewart-Brown SL. Obesity and physical and emotional well-being: associations between body mass index, chronic illness, and the physical and mental components of the SF-36 questionnaire. Obes Res 2000; 8:160-70.

22. Almeida GAN, Loureiro SR, Santos JE. Obesidade mórbida em mulheres - estilos alimentares e qualidade de vida. Arch Latinoam Nutr 2001; 51:359-65.

23. Sarlio-Lähteenkorva S, Stunkard A, Rissanen A. Psychosocial factors and quality of life in obesity. Int J Obes Relat Metab Disord 1995; 19 Suppl 6:S1-5.

24. Richards MM, Adams TD, Hunt SC. Functional status and emotional well-being, dietary intake, and physical activity of severely obese subjects. J Am Diet Assoc 2000; 100:67-75.

25. Wadden TA, Stunkard AJ. Social and psychological consequences of obesity. Ann Intern Med 1985; 103:1062-7.

26. Andrade G. Grupo de apoio social no hospital: o caso do "lutando para viver" [Dissertação de Mestrado]. Rio de Janeiro: Escola Nacional de Saúde Pública, Fundação Oswaldo Cruz; 2001.

27. Shaikh AR, Yaroch AL, Nebeling L, Yeh MC, Resnicow K. Psychosocial predictors of fruit and vegetable consumption in adults: a review of the literature. Am J Prev Med 2008; 34:535-43.

28. Schmidt MI, Tavares DB, Polanczyk M, Palta M, Prineas RJ, Berman R, et al. Comparison of selfreported and measured height and weight. Am J Epidemiol 1982; 115:223-30.

29. Spencer EA, Appleby PN, Davey GK, Key TJ. Validity of self-reported height and weight in $4808 \mathrm{EP}-$ IC-Oxford participants. Public Health Nutr 2002; 5:561-5.

30. Nyholm M, Gullberg B, Merlo J, Lundqvist-Persson C, Råstam L, Lindblad U. The validity of obesity based on self-reported weight and height: implications for population studies. Obesity (Silver Spring) 2007; 15:197-208.

31. Silveira EA, Araújo CL, Gigante DP, Barros AJ, Lima MS. Validação do peso e altura referidos para o diagnóstico do estado nutricional em uma população de adultos no Sul do Brasil. Cad Saúde Pública 2005; 21:235-45.

32. Rothman KJ. Epidemiology: an introduction. Oxford: Oxford University Press; 2002.

Recebido em 17/Ago/2009

Versão final reapresentada em 30/Nov/2010 Aprovado em 18/Jan/2011 